\title{
GAIA Level 4 Small for Gestational Age
}

National Cancer Institute

\section{Source}

National Cancer Institute. GAIA Level 4 Small for Gestational Age. NCI Thesaurus. Code C128738.

GAIA Level 4 Small for Gestational Age is defined by one of the following three criteria: first, the infant is noted to be small, but has no documented weight; second, the gestational age is assessed by infant examination only; third, the diagnosis is extracted from either billing codes or a chart with no documentation of the actual birth weight or the gestational age. 